\title{
Bifurcation Solutions of Non-linear Fourth Order Ordinary Differential Equation with Two Parameters
}

\author{
Mudhir A. Abdul Hussain \\ (Communicated by İ. Onur KIYMAZ)
}

\begin{abstract}
In this article we are interested in the study of bifurcation solutions of non-linear fourth order differential equation by using local method of Lyapunov -Schmidt. The reduced equation corresponding to the non-linear fourth order differential equation is given by a nonlinear system of two algebraic equations. The classification of the solutions (equilibrium points) of this system has been discussed.
\end{abstract}

Keywords: Bifurcation theory; Nonlinear systems; Local Lyapunov-Schmidt method.

AMS Subject Classification (2010): Secondary: 34K18; 93C10.

\section{Introduction} form

Many of the nonlinear problems that appear in Mathematics and Physics can be written in the operator equation

$$
F(x, \lambda)=b, \quad x \in O \subset X, \quad b \in Y, \quad \lambda \in R^{n}
$$

where $F$ is a smooth Fredholm map of index zero and $X, Y$ are Banach spaces and $O$ is open subset of $X$. For these problems, the method of reduction to finite dimensional equation,

$$
\theta(\xi, \lambda)=\tilde{\beta}, \quad \xi \in \tilde{M}, \quad \tilde{\beta} \in \tilde{N} .
$$

can be used, where $\tilde{M}$ and $\tilde{N}$ are smooth finite dimensional manifolds. A passage from (1.1) into (1.2) (variant local scheme of Lyapunov -Schmidt) with the conditions that equation (1.2) has all the topological and analytical properties of (1.1) (multiplicity, bifurcation diagram, etc) can be found in [11-14]. Vainberg [11], Loginov [3] and Sapronov [13,14] are dealing with equation (1.1) into equation (1.2) by using local method of Lyapunov -Schmidt with the conditions that, equation (1.2) has all the topological and analytical properties of equation (1.1) ( multiplicity, bifurcation diagram, etc). The non-linear fourth order ordinary differential equation can be written in the following general form

$$
\begin{array}{r}
\frac{d^{4} w}{d x^{4}}+\alpha \frac{d^{2} w}{d x^{2}}+\beta w+g(\lambda, \tilde{w})=0, \\
\tilde{w}=\left(w, w^{\prime}, w^{\prime \prime}, w^{\prime \prime \prime}, w^{\prime \prime \prime \prime}\right) .
\end{array}
$$

where $\alpha, \beta$ are parameters and $g(\lambda, \tilde{w})$ is the nonlinear part. There are many studies of non-linear fourth order ordinary differential equations in different ways. In [4] Thompson and Stewart showed numerically the existence of 
periodic solutions of equation (1.3) for some values of parameters. Bardin and Furta in [1] used the local method of Lyapunov-Schmidt and found the sufficient conditions of existence of periodic waves of equation (1.3), also they are introduced the solutions of equation (1.3) in the form of power series. Sapronov Yu.I [13-14] applied the local method of Lyapunov-Schmidt and found the bifurcation solutions of equation (1.3). Abdul Hussain [5-8] applied the local method of Lyapunov-Schmidt and studied equation (1.3) with different nonlinear parts. He discussed the bifurcation solutions of the reduced equation corresponding to the equation (1.3). In [9-10] Abdul Hussain used the modify Lyapunov-Schmidt method to study equation (1.3) by finding the nonlinear Ritz approximation correspondence to the equation (1.3).

In this paper we used the local method of Lyapunov-Schmidt to study the bifurcation solutions of boundary value problem,

$$
\begin{gathered}
\frac{d^{4} w}{d x^{4}}+\alpha \frac{d^{2} w}{d x^{2}}+\beta w+w w^{\prime \prime}+\left(w^{\prime}\right)^{2}=0 \\
w(0)=w(1)=w^{\prime \prime}(0)=w^{\prime \prime}(1)=0
\end{gathered}
$$

\section{Reduction to Bifurcation Equation.}

The following theorem and definition are important in our work:

Theorem 2.1 Suppose $X$ and $Y$ are real Banach spaces $F(x, \lambda)$ and is a $C^{1}$ map defined in a neighborhood $U$ of a point $\left(x_{0}, \lambda_{0}\right)$ with range in $\mathrm{Y}$ such that $F\left(x_{0}, \lambda_{0}\right)=0$ and $F_{x}\left(x_{0}, \lambda_{0}\right)$ is a linear Fredholm operator. Then all solutions $(x, \lambda)$ of $F(x, \lambda)=0$ near $\left(x_{0}, \lambda_{0}\right)$ (with $\lambda$ fixed) are in one-to-one correspondence with the solutions of a finite-dimensional system of $N_{1}$ real equations in a finite number $N_{0}$ of real variables. Furthermore, $N_{0}=\operatorname{dim}(\operatorname{ker}(L))$ and $N_{1}=\operatorname{dim}(\operatorname{coker}(L)),\left(L=F_{x}\left(x_{0}, \lambda_{0}\right)\right)$.

Definition 2.1 The Discriminate set $\Sigma$ of equation (1.1) is defined to be the union of all $\lambda=\bar{\lambda}$ for which the equation (1.1) has degenerate solution $\bar{x} \in O$ :

$$
F(\bar{x}, \bar{\lambda})=b, \quad \operatorname{codim}\left(\operatorname{Im} \frac{\partial F}{\partial x}(\bar{x}, \bar{\lambda})\right)>0
$$

where, $I m$ - is the image of the operator $\frac{\partial F}{\partial x}(\bar{x}, \bar{\lambda})$.

To the study problem(1.4) it is convenient to set the ODE in the form of operator equation, that is;

$$
F(w, \lambda)=\frac{d^{4} w}{d x^{4}}+\alpha \frac{d^{2} w}{d x^{2}}+\beta w+w w^{\prime \prime}+\left(w^{\prime}\right)^{2}
$$

Where $F: E \rightarrow M$ is nonlinear Fredholm map of index zero from Banach space $E$ to Banach space $M, E=$ $C^{4}([0,1], R)$ is the space of all continuous functions that have derivative of order at most four, $M=C^{0}([0,1], R)$ is the space of all continuous functions and $w=w(x), x \in[0,1], \lambda=(\alpha, \beta)$. In this case, the bifurcation solutions of equation (2.1) is equivalent to the bifurcation solutions of operator equation

$$
F(w, \lambda)=0
$$

It is well known that by finite dimensional reduction theorem (1) the solutions of problem (1.4) is equivalent to the solutions of finite dimensional system with $2=\operatorname{dim}\left(\operatorname{ker} F_{w}(0, \lambda)\right)$ variables and $2=\operatorname{dim}\left(\operatorname{coker} F_{w}(0, \lambda)\right)$ equations, so we will find this system and then we analyze the results to study the bifurcation solutions of problem (1.4). The first step in this reduction is to determines the linearized equation corresponding to the equation (2.2), which is given by the following equation,

$$
\begin{gathered}
A h=0, \quad h \in E \\
A=\frac{\partial F}{\partial w}(0, \lambda)=\frac{d^{4}}{d x^{4}}+\alpha \frac{d^{2}}{d x^{2}}+\beta, \quad x \in[0,1], \\
h(0)=h(1)=h^{\prime \prime}(0)=h^{\prime \prime}(1)=0
\end{gathered}
$$


The solution of linearized equation which is satisfied the boundary conditions is given by,

$$
e_{p}=c_{p} \sin (p \pi x), \quad p=1,2,3, \ldots
$$

and the characteristic equation corresponding to this solution is,

$$
p^{4} \pi^{4}-\alpha p^{2} \pi^{2}+\beta=0
$$

This equation gives in the $\alpha \beta$-plane characteristic lines $l_{p}$. The characteristic lines $l_{p}$ consist the points $(\alpha, \beta)$ in which the linearized equation has non-zero solutions. The point of intersection of characteristic lines in the $\alpha \beta$-plane is a bifurcation point [14]. So for equation (2.2) the point $(\alpha, \beta)=\left(5 \pi^{2}, 4 \pi^{4}\right)$ is a bifurcation point. Localized parameters $\alpha, \beta$ as follows,

$$
\alpha=5 \pi^{2}+\delta_{1}, \quad \beta=4 \pi^{4}+\delta_{2}, \quad \delta_{1}, \delta_{2} \text { are small parameters } .
$$

lead to bifurcation along the modes

$$
e_{1}(x)=c_{1} \sin (\pi x), \quad e_{2}(x)=c_{2} \sin (2 \pi x)
$$

where $\left\|e_{1}\right\|_{H}=\left\|e_{2}\right\|_{H}=1$ and $c_{1}=c_{2}=\sqrt{2},\left(H\right.$ is a Hilbert space $\left.L^{2}([0,1], R)\right)$. Let $N=\operatorname{ker}(A)=\operatorname{span}\left\{e_{1}, e_{2}\right\}$, then the space $E$ can be decomposed in direct sum of two subspaces, $N$ and the orthogonal complement to $N$,

$$
E=N \oplus N^{\perp}, \quad N^{\perp}=\{v \in E: v \perp N\} .
$$

Similarly, the space $M$ can be decomposed in direct sum of two subspaces, $N$ and the orthogonal complement to $N$,

$$
M=N \oplus \tilde{N}^{\perp}, \quad \tilde{N}^{\perp}=\{v \in M: v \perp N\} .
$$

There exist two projections $P: E \rightarrow N$ and $I-P: E \rightarrow N^{\perp}$ such that $P w=u,(I-P) w=v$ and hence every vector $w \in E$ can be written in the form of

$$
w=u+v, \quad u=\sum_{i=1}^{2} \xi_{i} e_{i} \in N, \quad v \in N^{\perp}, \quad \xi_{i}=\left\langle w, e_{i}\right\rangle_{H} .
$$

Similarly, there exists projections $Q: M \rightarrow N$ and $I-Q: M \rightarrow \tilde{N}^{\perp}$ such that

$$
\begin{gathered}
Q F(w, \lambda)=F_{1}(w, \lambda), \\
(I-Q) F(w, \lambda)=F_{2}(w, \lambda),
\end{gathered}
$$

and hence,

$$
\begin{gathered}
F(w, \lambda)=F_{1}(w, \lambda)+F_{2}(w, \lambda), \\
F_{1}(w, \lambda)=\sum_{i=1}^{2} v_{i}(w, \lambda) e_{i} \in N, \quad F_{2}(w, \lambda) \in \tilde{N}^{\perp}, \\
v_{i}(w, \lambda)=\left\langle F(w, \lambda), e_{i}\right\rangle_{H} .
\end{gathered}
$$

Accordingly, equation (2.2) can be written in the form of

$$
\begin{gathered}
Q F(w, \lambda)=0, \\
(I-Q) F(w, \lambda)=0
\end{gathered}
$$

or ,

$$
\begin{gathered}
Q F(u+v, \lambda)=0, \\
(I-Q) F(u+v, \lambda)=0
\end{gathered}
$$

By implicit function theorem, there exists a smooth map $\Phi: N \rightarrow N^{\perp}$ (depending on $\lambda$ ), such that, $\Phi(u, \lambda)=v$ and

$$
(I-Q) F(u+\Phi(u, \lambda), \lambda)=0
$$


To find the solutions of the equation $F(w, \lambda)=0$ in the neighbourhood of the point $w=0$ it is sufficient to find the solutions of the equation,

$$
Q F(u+\Phi(u, \lambda), \lambda)=0
$$

Equation (2.3) is called bifurcation equation of the equation (2.2). Hence we have the bifurcation equation in the form of

$$
\Theta(\xi, \lambda)=0, \quad \xi=\left(\xi_{1}, \xi_{2}\right), \quad \lambda=(\alpha, \beta) .
$$

where,

$$
\Theta(\xi, \lambda)=F_{1}(u+\Phi(u, \lambda), \lambda)
$$

Equation (2.2) can be written in the form of

$$
\begin{aligned}
F(u+v, \lambda) & =A(u+v)+T(u+v) \\
& =A u+u u^{\prime \prime}+\left(u^{\prime}\right)^{2}+\ldots
\end{aligned}
$$

where, $T(u+v)=(u+v)(u+v)^{\prime \prime}+\left((u+v)^{\prime}\right)^{2}$ and the dots denote the terms consists the element $v$. Since,

$$
\Theta(\xi, \lambda)=F_{1}(u+v, \lambda)=0 .
$$

we get from equation (2.4)

$$
\sum_{i=1}^{2}\left\langle A u+u u^{\prime \prime}+\left(u^{\prime}\right)^{2}, e_{i}\right\rangle_{H} e_{i}+\ldots=0
$$

After some calculations of equation (2.5) and by using the property $A e_{1}=\alpha_{1}(\lambda) e_{1}, \quad A e_{2}=\alpha_{2}(\lambda) e_{2}$ we have the following result,

$$
\left(A_{1} \xi_{1}^{2}+A_{2} \xi_{2}^{2}+A_{3} \xi_{1}\right) e_{1}+\left(B_{1} \xi_{1} \xi_{2}+B_{2} \xi_{2}\right) e_{2}=0
$$

where,

$$
\begin{gathered}
A_{1}=-\frac{4 \sqrt{2} \pi}{3}, \quad A_{2}=-\frac{16 \sqrt{2} \pi}{15}, \quad A_{3}=\lambda_{1}=\alpha_{1}(\lambda) \\
B_{1}=-\frac{128 \sqrt{2} \pi}{15}, \quad B_{2}=\lambda_{2}=\alpha_{2}(\lambda)
\end{gathered}
$$

$\alpha_{1}(\lambda), \alpha_{2}(\lambda)$ are smooth functions and

$$
\langle g(x), h(x)\rangle_{H}=\int_{0}^{1} g(x) h(x) d x
$$

Equation (2.6) can be written in the form of

$$
\begin{aligned}
A_{1} \xi_{1}^{2}+A_{2} \xi_{2}^{2}+A_{3} \xi_{1} & =0, \\
B_{1} \xi_{1} \xi_{2}+B_{2} \xi_{2} & =0 .
\end{aligned}
$$

It is easy to see that system (2.7) is equivalent to the following system

$$
\begin{aligned}
-\xi_{1}^{2}-\xi_{2}^{2}-q_{1} \xi_{1} & =0, \\
-2 \xi_{1} \xi_{2}-q_{2} \xi_{2} & =0 .
\end{aligned}
$$

where $q_{1}$ and $q_{2}$ are real parameters. From the above results we conclude the following theorem,

Theorem 2.2 The bifurcation equation

$$
\Theta(\xi, \lambda)=F_{1}(u+\Phi(u, \lambda), \lambda)=0
$$


corresponding to the equation (2.2) have the following form,

$$
\Theta(\xi, \tilde{\lambda})=\left(\begin{array}{c}
-\xi_{1}^{2}-\xi_{2}^{2}-q_{1} \xi_{1} \\
-2 \xi_{1} \xi_{2}-q_{2} \xi_{2}
\end{array}\right)+o\left(|\xi|^{2}\right)+O\left(|\xi|^{2}\right) O(\delta)=0
$$

where, $\xi=\left(\xi_{1}, \xi_{2}\right),\left(q_{1}, q_{2}\right)=\tilde{\lambda} \in R^{2}, \quad \delta=\left(\delta_{1}, \delta_{2}\right)$.

We note that system (2.8) is contact equivalence to the system (2.9). From theorem (2.1) the solutions of equation (2.2) are in one-to-one correspondence with the solutions of system (2.8). Also, system (2.8) has all the topological and analytical properties of equation (2.2). Thus to study the bifurcation solutions of equation (2.2) it is sufficient to study the bifurcation solutions of system (2.8).

\section{Bifurcation Analysis of system (2.8)}

From section (2) we have that the point $a \in E$ is a solution of equation (2.2) if and only if

$$
a=\sum_{i=1}^{2} \bar{\eta}_{i} e_{i}+\Phi(\bar{\eta}, \bar{\lambda}) .
$$

where, $\bar{\eta}$ is a solution of system (2.8) and the discriminant set of equation (2.2) is equivalent to the discriminant set of system (2.8). It is noted that the solutions of system (2.8) can be classified as an equilibrium points of the dynamical system

$$
\begin{aligned}
& \dot{\xi_{1}}=-\xi_{1}^{2}-\xi_{2}^{2}-q_{1} \xi_{1}, \\
& \dot{\xi_{2}}=-2 \xi_{1} \xi_{2}-q_{2} \xi_{2} .
\end{aligned}
$$

so that the behavior of system (2.8) near the regular solutions is similar to the behavior of the dynamical system (3.1 ) near the equilibrium points. The system (3.1) is a gradient vector field

$$
\begin{array}{r}
\dot{\xi_{1}}=-\frac{\partial V}{\partial \xi_{1}}, \quad \dot{\xi}_{2}=-\frac{\partial V}{\partial \xi_{2}}, \\
V\left(\xi_{1}, \xi_{2}, \tilde{\lambda}\right)=\frac{\xi_{1}^{3}}{3}+\xi_{1} \xi_{2}^{2}+\frac{q_{1}}{2} \xi_{1}^{2}+\frac{q_{2}}{2} \xi_{2}^{2} .
\end{array}
$$

The solutions (equilibrium points) of the system (3.1) are

$$
p_{1}=(0,0), \quad p_{2}=\left(-q_{1}, 0\right), \quad p_{3,4}=\left(-\frac{q_{2}}{2}, \pm \frac{1}{2} \sqrt{2 q_{1} q_{2}-q_{2}^{2}}\right) .
$$

These solutions are degenerate on the lines $q_{1}=0, q_{2}=0$ and $q_{2}=2 q_{1}$. It follows that the Discriminant set $\Sigma$ of equations (2.8) in the space of parameters $\left(q_{1}, q_{2}\right)$ is given by the equation

$$
q_{1} q_{2}\left(2 q_{1}-q_{2}\right)=0
$$

The remaining solutions (equilibrium points) of system (3.1) not lies on the lines $q_{1}=0, q_{2}=0$ and $q_{2}=2 q_{1}$ are nondegenerate.

Since, $u=\sum_{i=1}^{2} \xi_{i} e_{i}$ we get from theorem (2.1) the solution $p_{1}=(0,0)$ correspondence to the solution $u_{1}=0$, the solution $p_{2}=\left(-q_{1}, 0\right)$ correspondence to the solution $u_{2}=-q_{1} e_{1}$ and the solutions $p_{3,4}=\left(-\frac{q_{2}}{2}, \pm \frac{1}{2} \sqrt{2 q_{1} q_{2}-q_{2}{ }^{2}}\right)$ correspondence to the solutions $u_{3,4}=-\frac{q_{2}}{2} e_{1} \pm \frac{1}{2} \sqrt{2 q_{1} q_{2}-q_{2}^{2}} e_{2}$. Let

$$
\begin{array}{lll}
R_{1}=\left\{\left(q_{1}, q_{2}\right): q_{2}>2 q_{1}>0\right\}, & R_{2}=\left\{\left(q_{1}, q_{2}\right): 2 q_{1}>q_{2}>0\right\}, \\
R_{3}=\left\{\left(q_{1}, q_{2}\right): 2 q_{1}>0>q_{2}\right\}, & R_{4}=\left\{\left(q_{1}, q_{2}\right):\right. & \left.q_{2}<2 q_{1}<0\right\}, \\
R_{5}=\left\{\left(q_{1}, q_{2}\right): 2 q_{1}<q_{2}<0\right\}, & R_{6}=\left\{\left(q_{1}, q_{2}\right):\right. & \left.q_{2}>0>2 q_{1}\right\} .
\end{array}
$$

then we can classify the equilibrium points of system (3.1) as follows: 
1. The point $p_{1}=(0,0)$ : At this point the eigenvalues of the jacobian matrix of system (3.1) are $-q_{1}$ and $-q_{2}$. It follows that the point $p_{1}$ is a stable (sink) in the sets $R_{1}$ and $R_{2}$. In the sets $R_{4}$ and $R_{5}$ is unstable (source). In the sets $R_{3}$ and $R_{6}$ is unstable (saddle).

2. The point $p_{2}=\left(-q_{1}, 0\right)$ : At this point the eigenvalues of the jacobian matrix of system (3.1) are $q_{1}$ and $2 q_{1}-q_{2}$. It follows that the point $p_{2}$ is unstable (saddle) in the sets $R_{1}, R_{4}$. In the regions $R_{5}$ and $R_{6}$ is a stable (sink). In the sets $R_{2}$ and $R_{3}$ is unstable (source).

3. The points $p_{3,4}=\left(-\frac{q_{2}}{2}, \pm \frac{1}{2} \sqrt{2 q_{1} q_{2}-q_{2}^{2}}\right)$ : At these points the eigenvalues of the jacobian matrix of system (3.1) are $\frac{q_{2}}{2}-\frac{q_{1}}{2} \pm \frac{\sqrt{\triangle}}{2}, \triangle=-3 q_{2}^{2}+6 q_{1} q_{2}+q_{1}^{2}$. The points $p_{3,4}$ are real only in the sets $R_{2}$ and $R_{5}$, so we will determine the type of these points in these sets. Since the determinant of the jacobian matrix of system (3.1) at these points is $q_{2}\left(q_{2}-2 q_{1}\right)$, it follows that from the definitions of the sets $R_{2}$ and $R_{5}$ the value of the determinant is negative and the value of $\triangle$ is positive this implies that the points $p_{3,4}$ are unstable (saddle) in both sets.

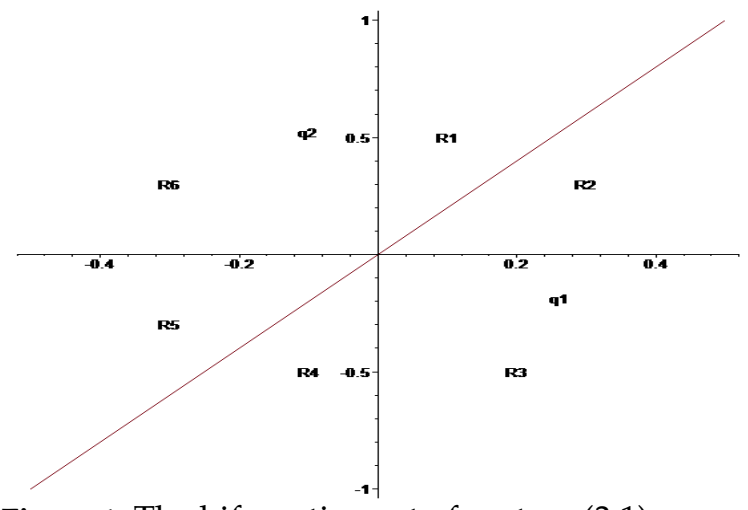

Figure 1. The bifurcation set of system (3.1).
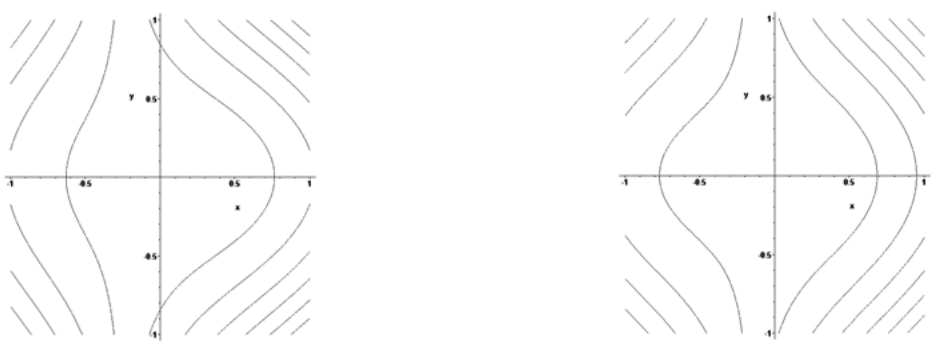

Figure 2. The level curves of the function (3.2) in the sets $R_{1}$ (left) and $R_{2}$ (right). 

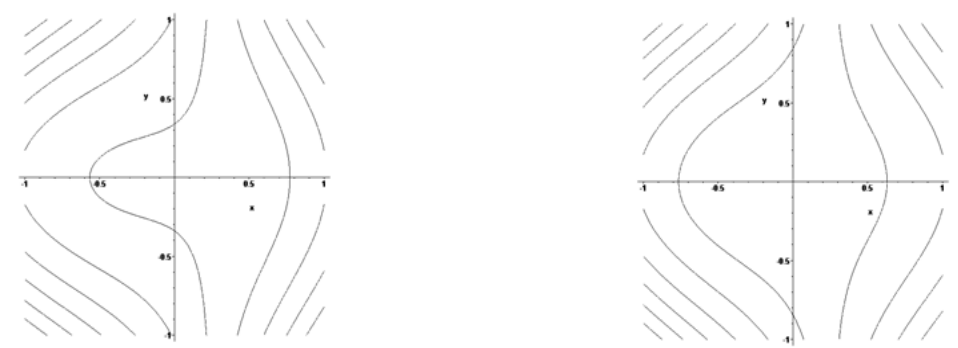

Figure 3. The level curves of the function (3.2) in the sets $R_{3}$ (left) and $R_{4}$ (right).
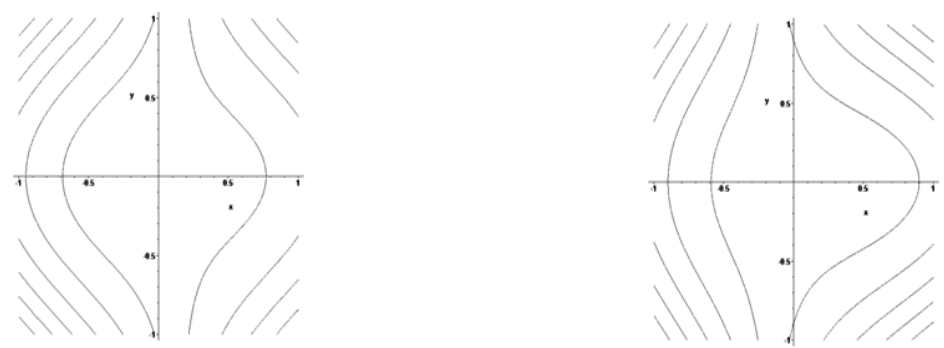

Figure 4. The level curves of the function (3.2) in the sets $R_{5}$ (left) and $R_{6}$ (right).

Acknowledgements I would like to thank the referee for useful suggestions and for his comments. 


\section{References}

[1] B. S. Bardin and S. D. Furta, Local existence theory for periodic wave moving of an infinite beam on a nonlinearly elastic support, Actual Problems of Classical and Celestial Mechanics, Elf, Moscow, 13-22 (1998).

[2] B.M. Darinskii, C.L. Tcarev, Yu.I. Sapronov, Bifurcations of Extremals of Fredholm Functionals,Journal of Mathematical Sciences, Vol. 145, No. 6, 2007.

[3] B.V. Loginov, Theory of Branching Nonlinear Equations in Theconditions of Invariance Group, - Tashkent: Fan, 1985.

[4] J. M. T. Thompson and H. B. Stewart, Nonlinear Dynamics and Chaos, Chichester, Singapore, J. Wiley and Sons, 1986. V.R. M.A. Abdul Hussain, Corner Singularities of Smooth Functions in the Analysis of Bifurcations Balance of the Elastic Beams and Periodic Waves, Ph. D. thesis, Voronezh- Russia. 2005.

[5] M.A. Abdul Hussain,Bifurcation Solutions of Boundary Value Problem, Journal of Vestnik Voronezh, Voronezh State University, No. 1, 2007, 162-166, Russia.

[6] M.A. Abdul Hussain, Bifurcation Solutions of Elastic Beams Equation with Small Perturbation, Int. J. Math. Anal. (Ruse) 3 (18) (2009), 879-888.

[7] M.A. Abdul Hussain, Two Modes Bifurcation Solutions of Elastic Beams Equation with Nonlinear Approximation, Communications in Mathematics and Applications journal, Vol. 1, no. 2, 2010, 123-131. India.

[8] M.A. Abdul Hussain, Two-Mode Bifurcation in Solution of a Perturbed Nonlinear Fourth Order Differential Equation, Archivum Mathematicum (BRNO), Tomus 48 (2012), 27-37, Czech Republic.

[9] M.A. Abdul Hussain, A Method for Finding Nonlinear Approximation of Bifurcation Solutions of Some Nonlinear Differential Equations, Journal of Applied Mathematics and Bioinformatics, vol.3, no.3, 2013, UK.

[10] M.A. Abdul Hussain, Nonlinear Ritz Approximation for Fredholm Functionals, Electronic Journal of Differential Equations, Vol. 2015, No. 294,USA,2015, 1-11.

[11] M.M. Vainberg, V.A. Trenogin, Theory of Branching Solutions of Nonlinear Equations, M.-Science , 1969.

[12] Yu.I. Sapronov, Regular Perturbation of Fredholm Maps and Theorem about odd Field, Works Dept. of Math., Voronezh Univ., 1973. V. 10, 82-88.

[13] Yu.I. Sapronov, Finite Dimensional Reduction in the Smooth Extremely Problems, - Uspehi math., Science, 1996, V. 51, No. 1., 101- 132.

[14] Yu.I. Sapronov and V.R. Zachepa, Local Analysis of Fredholm Equation, Voronezh Univ., 2002.

\section{Affiliations}

Mudhir A. AbDul Hussain

ADDRESS: Basrah University, Dept. of Mathematics, College of Education for Pure Sciences, Basrah, IRAQ.

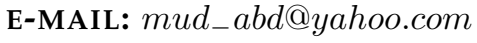

ORCID ID: $0000-0002-4730-6942$ 